\title{
- A Clinical Guide for Vertical Positioning of Bracket
}

\section{Rathi Amey J' ${ }^{1}$ Ahmed S Fayyaz ${ }^{2}$, Kalladka Gautham ${ }^{3}$, Iyer Kavitha ${ }^{4}$}

\section{IJCRR}

Section: Healthcare

ISI Impact Factor

(2019-20): 1.628

IC Value (2019): 90.81

$\operatorname{SJIF}(2020)=7.893$

(c) (7) (8)

Copyright@IJCRR
'Assistant Professor, Department of Dentistry, MGM Medical College and Hospital, Aurangabad, Maharashtra, India; ${ }^{2}$ Honorary Professor, Department of Orthodontics and Dentofacial Orthopaedics, Sri Ramakrishna Dental College and Hospital, Coimbatore, Tamil Nadu, India; ${ }^{3}$ Associate Professor, Department of Orthodontics and Dentofacial Orthopaedics, Dayanand Sagar College of Dental Sciences Bangalore, Karnataka, India; ${ }^{4}$ Department of Orthodontics and Dentofacial Orthopaedics, Ragas Dental College and Hospital, Chennai, Tamil Nadu, India.

\section{ABSTRACT}

Introduction: Bonding in orthodontics is a major and important part of treatment and any error in this procedure creates an impact on the outcome of treatment, esthetics and function.

Aim: The aim is to provide a clinical guide for vertical positioning of the bracket.

Methodology: Brackets was positioned with help of an MBT Boone gauge on the right central incisor and checked with a bracket holder. This is reconfirmed with the MBT Boone gauge and vice versa with the bracket holder on the right central incisor.

Result: This clinical innovation with the use of bracket holding plier helps prevent and minimizes the error of vertical positioning of brackets measured from the incisal edge.

Conclusion: Bracket holding plier helps to prevent and minimizes error and it is an easy, convenient and simple method for bonding and reducing chair side time.

Key Words: Boone Gauge, Bracket holder, Bracket position, Bracket prescription, Esthetics, Orthodontic

\section{INTRODUCTION}

Clinicians often find it difficult and time-consuming to bracket positioning indirect bonding on consultation. Use of bracket positioner gauge on consultation becomes hectic. All the more the bonding takes time if there is severe crowding or highly/palatally/rotated teeth in the arch.

In Orthodontics as we know the position of the bracket plays an important role. The correct placement of the bracket is essential to finish treatment with an excellent occlusion and beautiful smile. Errors in bracket position could lead to an improper finish, hampered esthetics and altered function of a case.

Angle recommended that the ideal position to place the bracket should be at the Centre of the labial surface of the tooth. ${ }^{1}$ Andrews developed the straight-wire appliance and proposed that the brackets should be placed at the midpoint of the facial axis [FA] point, as the midpoint of all the clinical crowns are located on the same plane [Andrews Plane], it was felt that the FA point was readily and consistently located. ${ }^{2}$ Ricketts, and later Kalange advocated the use of marginal ridges to guide the vertical positioning of brackets and bands. ${ }^{3,4}$ McLaughlin et al., advocated the positioning of brackets at a measured distance from the incisal edge, with different vertical positions recommended for different sized tooth. 5,6

The following way is described which is easy, simple and convenient to use with the least error in the vertical positioning of bracket for a clinician.

This case report was done to check the clinical guide for vertical positioning of the bracket. The objective of this paper is to help position the bracket accurately in the vertical dimension, fasten bonding, reduce chairside time.

\section{MATERIAL AND METHOD}

It's a one time marking to be made on a Bracket holder with a flat end.

Corresponding Author:

Dr. Rathi Amey J, BDS, MDS, Assistant Professor, Department of Dentistry, MGM Medical College and Hospital, Aurangabad, Maharashtra, India; Email: ameyrathi18@gmail.com

ISSN: 2231-2196 (Print) ISSN: 0975-5241 (Online)

Received: $14.04 .2021 \quad$ Revised: 23.05 .2021

Accepted: 25.06 .2021

Published: 10.08 .2021 
Material required:

1. MBT Boone Gauge \{Clinician can use any gauge\}

2. Bracket Holder with the flat posterior end

3. Divider with Scale

4. Air-rotor handpiece with Long Tapered fissure bur

5. Dental model

6. Anterior brackets $[\mathrm{MBT}]$

\section{Step 1}

First, position the brackets with help of the MBT Boone gauge on the right central incisor and bond it.[fig. 1] Then measure the distance from the incisal edge to the lower border of the bracket with help of Vernier calliper or divider \{ to note that for canine, the tip is considered for measurement\}. These measurements will serve as guidelines for the vertical positioning of the brackets on anterior teeth. [fig. 2]

\section{Step 2}

Now transfer the measured distance from the divider to the flat end of the bracket holder, [fig. 3 a] and mark parallel lines on the posterior end of bracket holder with a gap of $0.5 \mathrm{~mm}$ each with help of divider and later groove those markings with air rotor and tapered fissure bur to prevent it from smudging.[fig. $\mathbf{3}$ b]. The markings are confirmed by placing the flat end of the bracket holder on the right central incisor.[fig 4]. The markings should be made for the position for Central [ X mm], Lateral [X-0.5mm], Canine [X mm]

Usually, when we place the bracket on central at $4.5 \mathrm{~mm}$ by MBT Boone gauge, the distance from incisal edge to bracket edge is $2.5 \mathrm{~mm}$, for laterals at $4 \mathrm{~mm}$ bracket placement corresponds to $2 \mathrm{~mm}$ from incisal edge to bracket edge and so on concerning canine as well as in posteriors too.

Start marking from $1.5 \mathrm{~mm}, 2 \mathrm{~mm}, 2.5 \mathrm{~mm}, 3 \mathrm{~mm}$ and $3.5 \mathrm{~mm}$ on the bracket holder on both sides. This gives an option to the operator to place brackets depending on the severity of the bite [ deep bite, open bite, normal bite].

\section{Step 3}

The next bracket is placed on the left central incisor and checked with a bracket holder with the grooved markings on the flat end for vertical height [fig 4]. This is reconfirmed with the MBT Boone gauge and vice versa with the bracket holder on the right central incisor. [fig $\mathbf{5 a}$, fig $\mathbf{5 b}$ respectively].

\section{RESULT}

The net result is the addition of an extra function to the already existing bracket holder i.e. the function of vertical positioning of bracket and reduction of an instrument from an Orthodontists consulting bag.

\section{DISCUSSION}

The above technique can be used as per the clinicians/operators choice of bracket placement, depending on which bracket prescription he/she follows [eg:- MBT versatile appliance bracket placement guide]. Fukuyo et al., digitized the models of 40 patients with normal occlusion and compared three methods of bracket placement (FA, height, and marginal ridge method). The bracket positions relative to a constructed virtual bracket plane were determined. They found that even if the brackets were positioned ideally for each technique, vertical errors will still count and therefore, suggested modifications to bracket positions for each technique.

Incorrectly positioned brackets can render even the most customized prescription ineffective and increase treatment time and the number of archwire adjustments necessary. ${ }^{8}$ Placement of brackets in the positions determined by measuring the distance from the incisal edge appears to be more accurate in the vertical dimensions for anterior teeth. ${ }^{9}$

Special consideration has to be made while positioning on premolar and canine due to curvature of the buccal surface where while positioning the bracket, the bracket holder should be perpendicular to bracket. Also one should know the total width of the flat end of the bracket holder to divide it into scales.

Although this method only helps in preventing or minimizing the error in the position of brackets in vertical parameters, the operator has to be cautious and manage the mesiodistal positioning of brackets through years of experience gained by practice.

This technique has the following advantages;

1. A reduction in error in vertical positioning, which can be avoided due to better precision, reproducibility and predictability of the bracket bonding.

2. It allows a practical standardized bonding procedure

3. Avoids bracket repositioning for vertical errors and/or the introduction of excessive corrective wire bends

4. Reduces chair time and unnecessary discomfort to the patient

5. It's easy, simple, convenient and cheap.

\section{CONCLUSION}

Bracket holding plier helps to prevent and minimizes error and it is an easy, convenient and simple method for bonding and reducing chairside time. In general, this method of using bracket holder for positioning allows orthodontists to obtain adequate bracket positioning leading to a finer finish with fewer errors, good occlusion and better esthetics.

\section{Acknowledgement: Nil}

Financial support and sponsorship: Nil 
Conflicts of Interest: There are no conflicts of interest

\section{Authors contribution}

1. Dr. Amey J Rathi: design

2. Dr. S Fayyaz Ahmed: manuscript writing

3. Dr. Gautham Kalladka: editing

4. Dr.Kavitha Iyer: review

\section{REFERENCES}

1. Angle EH. The latest and best in orthodontics mechanism. Dental Cosmos. 1928; 70; 1143-1158

2. Andrews LF. The straight wire appliance: Origin, controversy, commentary. J Clin Orthod.1976; 10: 99-114

3. Ricketts MR. Big progressive therapy as an answer to orthodontic needs. American J Orthod. 1976; 70:241-267
4. Kalanga JT. Ideal appliance placement with APC brackets and indirect bonding. J Clin Orthod.1999; 33: 516-526

5. McLaughlin R P, Bennett J C. Bracket placement with the preadjusted appliance. J Clin Orthod. 1995; 29; 302-311

6. McLaughlin RP, Bennett JC. Finishing and pre-adjusted appliances. Seminar in Orthodontics.2003; 9; 165-183

7. Fukuyo K, Nishii Y, Nojima K, Yamaguchi H. A comparative study of three methods of bracket placement. Orthodontic Waves.2004; 63; 63-70

8. Carlson SK, Johnson E. Bracket positioning and resets: Five steps to align crowns and root consistently. American J Orthod Dentofac Orthoped. 2001; 119; 76-80

9. Armstrong D, Shen G, Petocz P, Darendellier MA. A comparison of accuracy in bracket positioning between two techniques- localizing the centre of the clinical crown and measuring the distance from the incisal edge. European J Orthod. 2007;29:430-436.

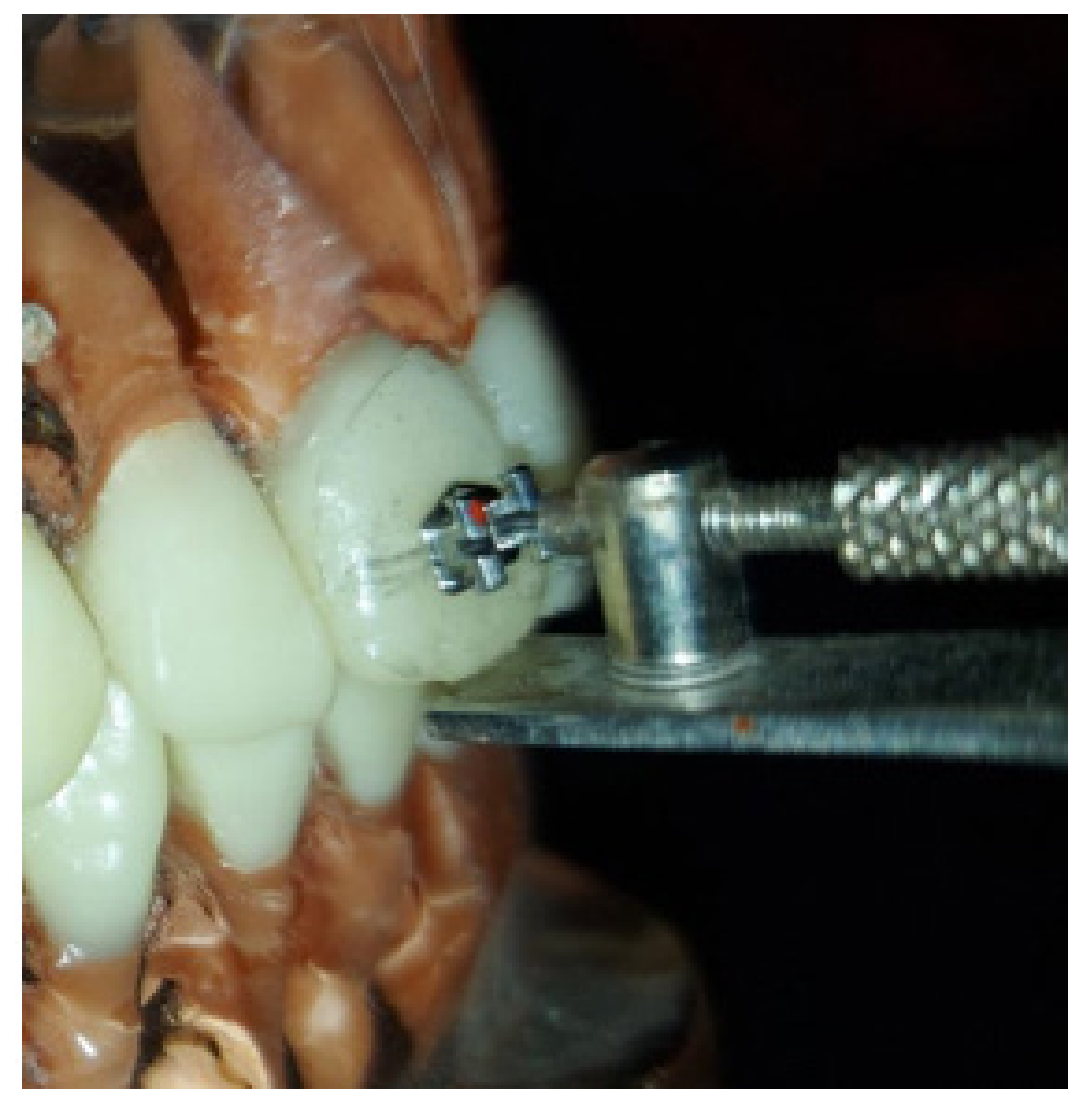

Figure 1: Bonding with help of Boone gauge 




Figure 2: Measuring the distance from the incisal edge by divider.



Figure 3a: Transferring the measurement to the flat end of bracket holder. The other marking is $1 \mathrm{~mm}$ shorter. 


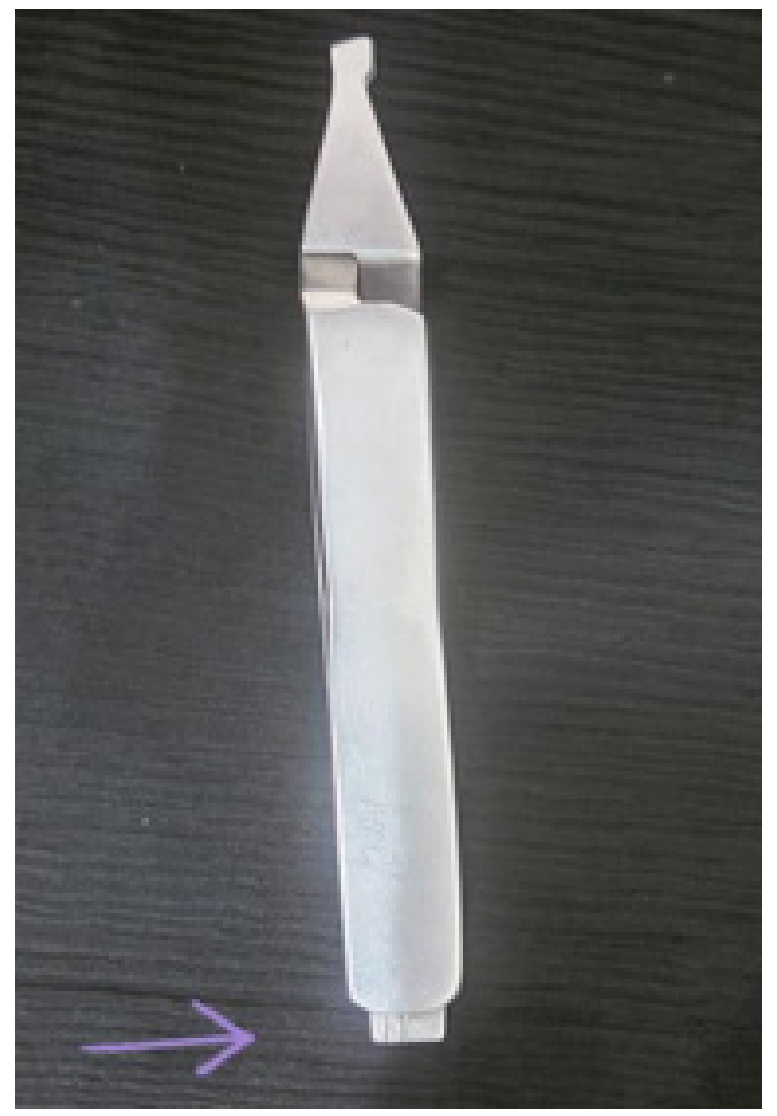

Figure 3b: Grooving of markings on bracket holder

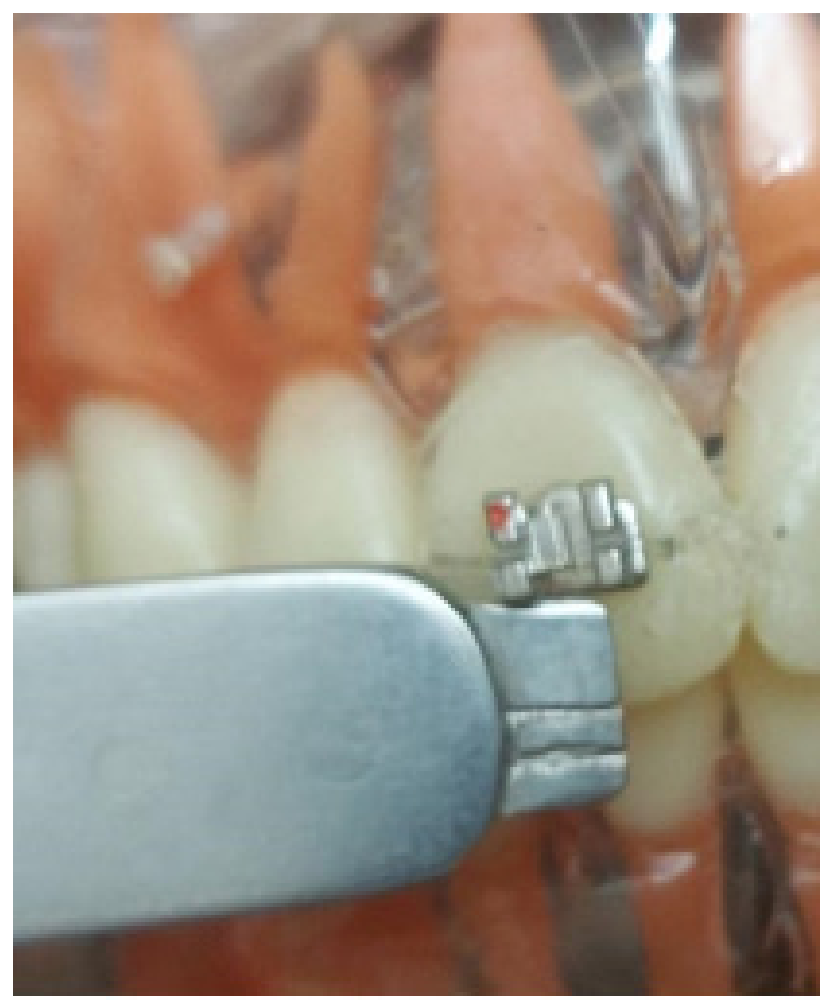

Figure 4: Confirming the markings on the flat end of bracket holder by placing on right central incisors which were positioned by Boone gauge 


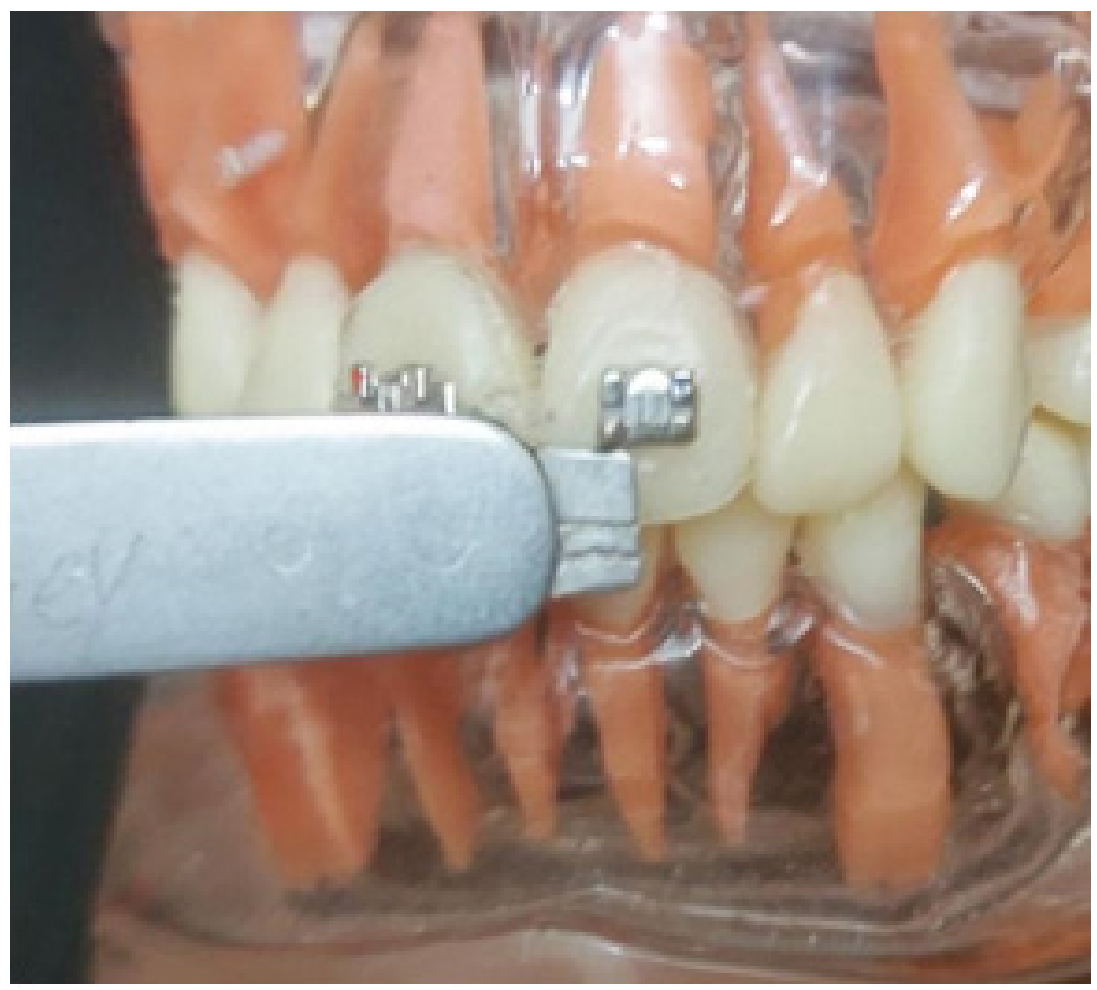

Figure 5a: Positioning bracket on left central incisor with help of bracket holder.

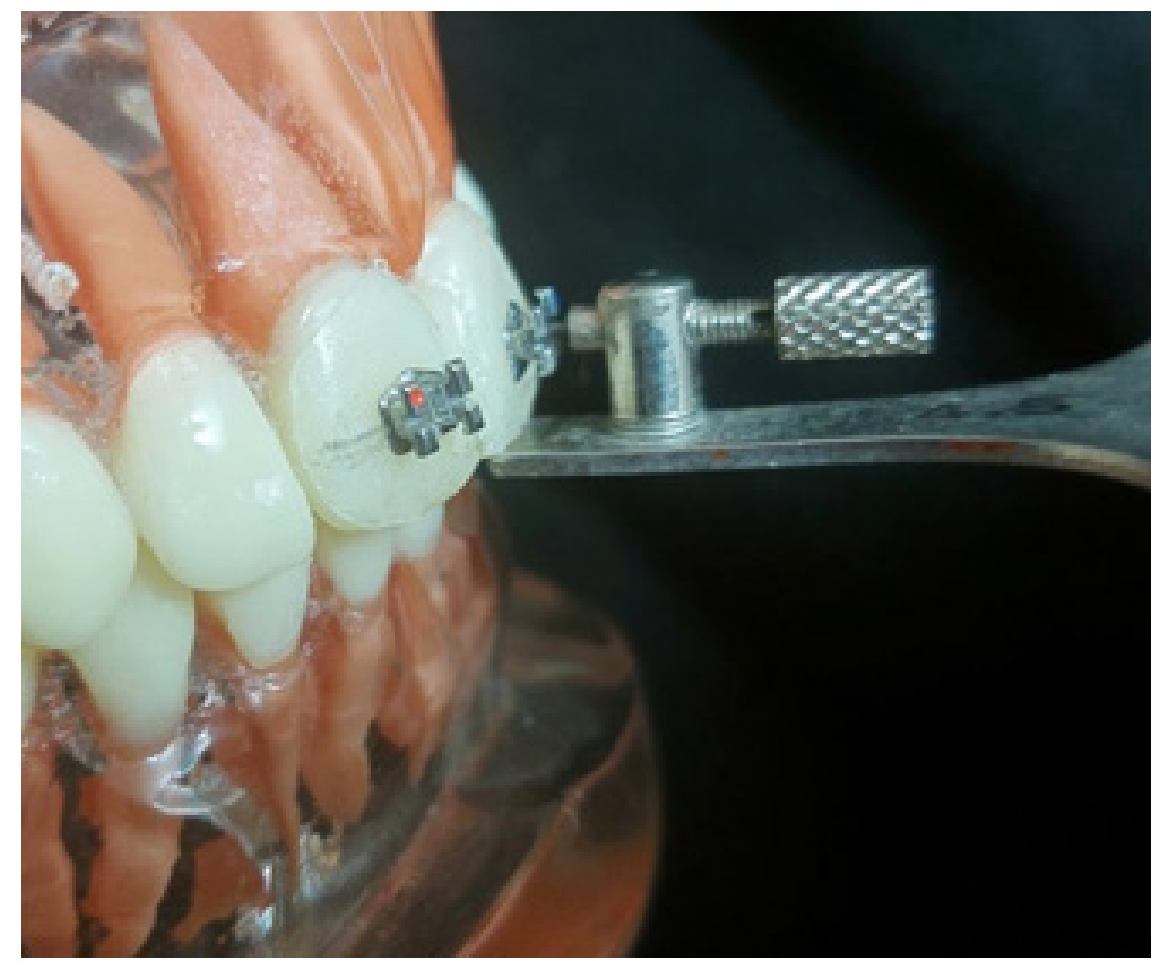

Figure 5b: Checking of vertical bracket position of left central incisor by Boone gauge. 